\title{
Heart-rate Monitoring System Design and Analysis Using a Nios II Soft-core Processor
}

\author{
Lim Chun Keat, Asral Bahari Jambek, and Uda Hashim
}

\begin{abstract}
The heart rate of a person is able to tell whether they are healthy. A heart-rate monitoring device is able to measure or record the heart rate of a person in real time, whether it is an electrocardiogram (ECG) or a photoplethysmogram (PPG). In this work, a microprocessor system loaded with a heart-rate monitoring algorithm is implemented. The microprocessor system is the Nios II processor system, which interfaces with an analogue-to-digital converter (ADC) and a pulse sensor. A beatfinding algorithm is used in the microprocessor system for heart rate measurement. An experiment is carried out to analyse the functionality of the microprocessor system loaded with the algorithm. The results show that the detected heart rate is in the range of the average human being's heart rate. The signal flow within the microprocessor system is observed and analysed using SignalTap II from Quartus' software. Based on a power analysis report, the proposed microprocessor system has a total power dissipation of around $218.26 \mathrm{~mW}$.
\end{abstract}

Keywords-Pulse sensor, PPG, Heart rate, FPGA, Nios II

\section{INTRODUCTION}

A heart-rate monitor is a device that measures or records the heart rate of a person in real time. A heart rate reveals the health condition of a person. This device performs noninvasive heart-rate monitoring by measuring physiological signals, using either an electrocardiogram (ECG) or a photoplethysmogram (PPG). Electrocardiography measures the electrical activities of the heart by using electrodes placed on the person's body for a given period of time. Photoplethysmography measures the change of volume within an organ or tissue such as the skin through an optical approach.

Photoplethysmography is currently widely used in heart-rate monitoring devices, especially portable devices. The PPG is used to calculate the heart rate and inter-beat interval. Some PPG-based systems are able detect the blood oxygen level (SpO2). Most PPG-based systems use a light-based sensor where the sensor mechanism detects the refraction and transmission of light. Certain PPG-based systems use more than one wavelength of light to improve the accuracy of the PPG, especially in pulse oximetry.

In this paper, a heart-rate measurement algorithm is implemented in a microprocessor system that interfaces with an ADC and a pulse sensor. Section II discusses several existing pulse-based acquisition systems, while Section III presents the methodology of implementing a microprocessor system loaded with a heart-rate measurement algorithm in a

This research was supported in part by Fundamental Research Grant Scheme, Ministry of Higher Education, Malaysia (FRGS Phase 1/2014).

Lim Chun Keat and Asral Bahari Jambek are with School of Microelectronic Engineering, Universiti Malaysia Perlis, Perlis, Malaysia, (email: wally.ck24@gmail.com, asral@unimap.edu.my).

Uda Hashim is with Insituite of Nano Electronic Engineering, Universiti Malaysia Perlis, Perlis, Malaysia, (e-mail: uda@unimap.edu.my).
Field Programmable Gate Array (FPGA) and the experimental set-up of a FPGA interfacing with an ADC and a pulse sensor. In Section IV, the experimental results obtained from the system are discussed. Finally, Section V concludes this paper.

\section{LITERATURE REVIEW}

In paper [1], a pulse oximetry system with motion artefact reduction based on Fourier analysis is introduced. Fig. 1 shows the block diagram of the proposed system, where the dotted boxes contain the sensor, the FPGA, the logic analyser (LA) and the personal computer (PC). In this system, a control signal is generated by the FPGA and sent to the PCB where analogue filtering is performed. In analogue filtering, the PCB receives the control signal from the FPGA and a signal from the photodiode that is converted from the light received from an LED. The signal is filtered and sent to the ADC where the signal will undergo digitalization. The digitalized signal is captured by a logic analyser and sent to the PC. Motion artefact reduction and $\mathrm{SpO}_{2}$ calculation will take place in the PC. The purpose of motion artefact reduction is to reduce the effect of noise caused by the motion artefact. The proposed system is used to calculate $\mathrm{SpO}_{2}$ or blood-oxygen levels, as well as to perform PPG- signal acquisition.

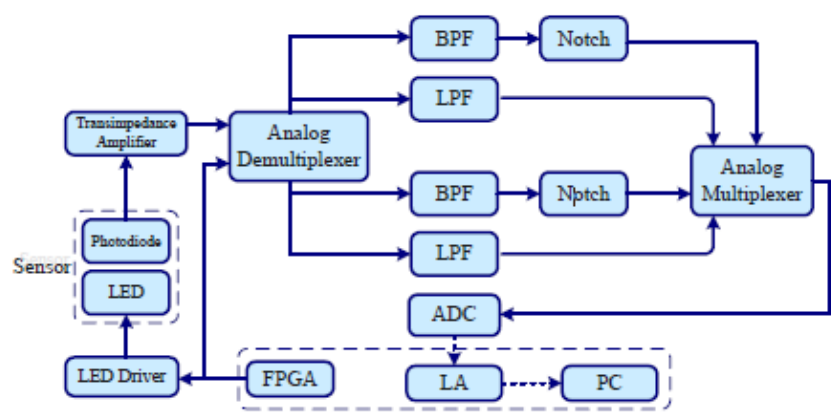

Fig. 1. Block diagram of the proposed pulse oximetry system [1].

The authors in paper [2] present a high-speed pulse data acquisition system based on an FPGA and a USB interface. The acquisition system consists of an HK-2000G pulse transducer, a Cyclone II series FPGA chip, an FTDI USB communication interface chip, a memory chip, an amplifier and filter circuits, a 16-bit A/D converter and a PC. Fig. 2 illustrates the block diagram of the pulse data acquisition system. The pulse signal is captured in real time from the sensor through the ADC. The signal is sampled by the ADC and stored in the PC's memory by the FPGA. The FPGA allows the data to be transferred to the PC through the USB interface, where the data is presented on the screen by using the PC's software. The system is developed to help doctors to analyse the pulse condition's information in real time. 


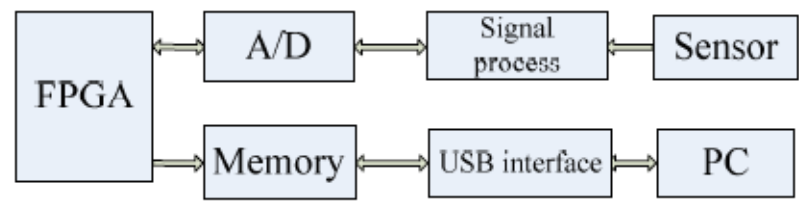

Fig. 2. Block diagram of the proposed pulse data acquisition system [2].

An FPGA-based Remote Pulse Rate Detection system is introduced by the authors in paper [3]. The proposed system uses the photoplethysmographic imaging method for pulse detection, where a low-cost digital camera is used as an image sensor for the system. A block diagram of the proposed system is illustrated in Fig. 3, showing the FPGA, the camera, an LCD and an SD card. The FPGA consists of a video pipeline and the proposed algorithm. The video pipeline carries out image processing from the camera. The proposed algorithm is a novel algorithm which is used for pulse rate measurement. In this proposed system, the pulse rate can be measured by placing a palm $20 \mathrm{~cm}$ away from the camera.

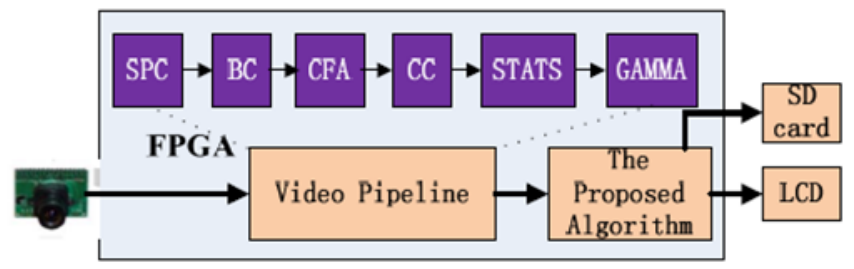

Fig. 3. Block diagram of proposed system [3].

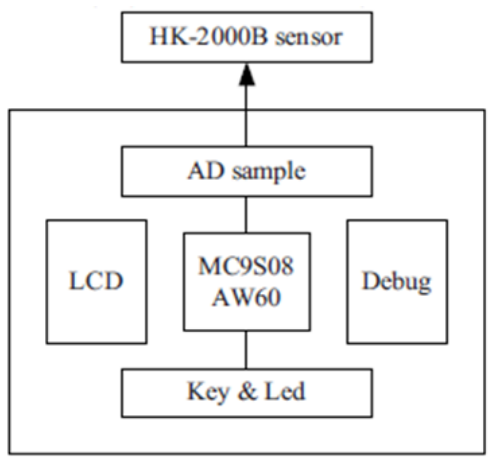

Fig. 4. Block diagram of the proposed pulse monitoring system [4].

In paper [4], a pulse monitoring system based on a feedback algorithm is introduced. In this system, a low power, 8-bit microcontroller with a built-in ADC is used. Besides the microcontroller, the system consists of an HK-2000B-type pulse sensor, an LCD display, LEDs, a Debug interface and buttons. The pulse monitoring system is illustrated in Fig. 4. A feedback algorithm is used in the microcontroller system to improve the detection accuracy of the pulse signal. The results show that the feedback algorithm does not reduce the accuracy of the system.

The authors in paper [5] implemented a pulse signal detection system with Bluetooth transmission. The structure of the system is illustrated in Fig. 5 and it consists of a pulse sensor, an Arduino microcontroller board, Bluetooth modules and a PC management platform. In the proposed system, the pulse signal is sampled by a built-in ADC of the Arduino microcontroller after the signal has been filtered and amplified. After the pulse signal is digitalized, data processing will be carried out on the pulse signal in the Arduino microcontroller board to calculate the pulse rate and time between heartbeats. The calculated results will be transmitted to the PC management platform though the Bluetooth modules. The PC management platform displays the calculated result and the pulse signal on the screen using processing software. Some of the related data are stored in the form of text file on the PC for further research

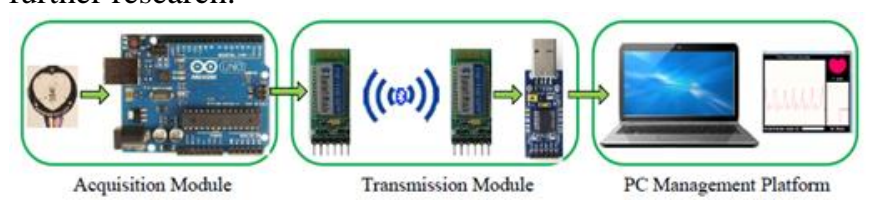

Fig. 5. Structure of the pulse signal detection system [5].

TABLE I

COMPARISON OF EXISTING MICROPROCESSOR SYSTEMS WITH AN ADC FOR BIOMEDICAL APPLICATION

\begin{tabular}{|l|c|c|c|c|c|}
\hline Reference & {$[1]$} & {$[2]$} & {$[3]$} & {$[4]$} & {$[5]$} \\
\hline System & PC & PC & $\begin{array}{c}\text { Dedicated } \\
\text { control block }\end{array}$ & 8-bit $\mu \mathrm{c}$ & $\begin{array}{c}\text { Arduino } \\
\mu \mathrm{c} \text { and PC }\end{array}$ \\
\hline ADC & Yes & 16-bit ADC & No & $\mu \mathrm{c}$ Built-in & $\mu \mathrm{c}$ Built-in \\
\hline Application & $\begin{array}{c}\text { Pulse } \\
\text { oximetry }\end{array}$ & $\begin{array}{c}\text { Pulse } \\
\text { analysis }\end{array}$ & $\begin{array}{c}\text { Remote pulse } \\
\text { rate } \\
\text { measurement }\end{array}$ & $\begin{array}{c}\text { Pulse } \\
\text { monitoring }\end{array}$ & $\begin{array}{c}\text { PPG } \\
\text { signal } \\
\text { analysis }\end{array}$ \\
\hline $\begin{array}{c}\text { Algorithm } \\
\text { calculation } \\
\text { and motion } \\
\text { artifact } \\
\text { reduction }\end{array}$ & $\begin{array}{c}\text { Huang } \\
\text { transform }\end{array}$ & $\begin{array}{c}\text { Image } \\
\text { processing } \\
\text { and novel } \\
\text { pulse rate } \\
\text { measurement } \\
\text { algorithm }\end{array}$ & $\begin{array}{c}\text { Feedback } \\
\text { algorithm }\end{array}$ & $\begin{array}{c}\text { Heart rate } \\
\text { and time } \\
\text { between } \\
\text { heartbeats } \\
\text { calculation }\end{array}$ \\
\hline Hardware & $\begin{array}{c}\text { FPGA, } \\
\text { ASIC, PC }\end{array}$ & $\begin{array}{c}\text { FPGA, } \\
\text { ASIC, PC }\end{array}$ & FPGA & ASIC & ASIC, PC \\
\hline
\end{tabular}

Several existing pulse-based acquisition systems are reviewed in this paper. The features of the existing systems are tabulated in Table I. Based on Table I, some of the systems used the PC as a main control or platform to execute the algorithm and display the results on the screen of the PC, while some of the acquisition systems are microcontroller-based systems. However, a custom design controller module is used in the acquisition system. Among the existing systems, most systems contain an ADC, such as a built-in ADC and an ADC chip. Pulse-based acquisition systems are normally able to detect pulse rates. However, some of the pulse-based acquisition systems are also able to detect blood oxygen levels. The algorithms of the systems are various, and the choice of algorithm depends on the designer of the system. The algorithm may include results' calculation, filtering and noise reduction. The choice of the system's hardware depends on the designer, and may integrate an FPGA or application-specific integrated circuit (ASIC), interface with a PC or have a combination of the two.

\section{METHODOLOGY}

In the previous section, the existing heart-rate monitoring systems are discussed; this section explains the algorithm for heart-rate monitoring and the proposed microprocessor system that interfaces with an ADC and a pulse sensor. The proposed system is a Nios II microprocessor system that is implemented on an FPGA to interface with the ADC and the pulse sensor. The system architecture consists of a Nios II economy version 
soft-core processor, two $32 \mathrm{Mb}$ SDRAMs with an SDRAM controller, a $32 \mathrm{~Kb}$ on-chip memory, a timer module, an Avalon bus, a Joint Test Action Group (JTAG), a universal asynchronous receiver transmitter (UART) and the general input and output (GPIO). Fig. 6 shows the block diagram of the proposed microprocessor system with an ADC and a pulse sensor. In this system, the interval period of the timer module is $500 \mu \mathrm{s}$. The ADC used for signal conversion between the pulse sensor and microprocessor system is an 8-bit microprocessor-compatible ADC integrated circuit (IC) from National Semiconductor, ADC0804. The ADC0804 is an 8-bit successive approximation ADC and has one differential analogue voltage input [6]. In the proposed system, ADC0804 receives an analogue input from the pulse sensor and provides an 8-bit digital output to the microprocessor system through the GPIO.

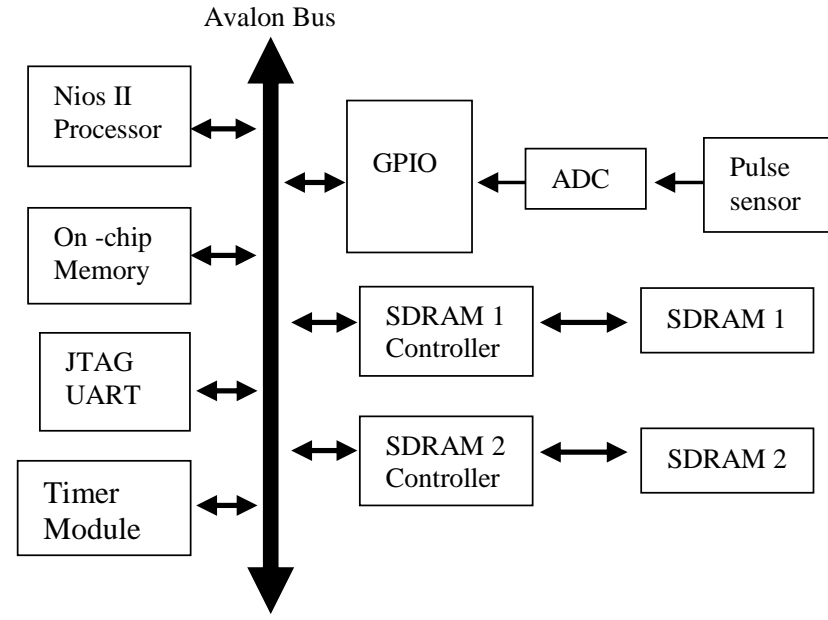

Fig. 6. Block diagram of a microprocessor system with an ADC and a pulse sensor.

In this work, a non-invasive heart-rate monitoring sensor is used, which is a PPG-based pulse sensor. The pulse sensor is an optical-based heart-rate sensor with the model name $\mathrm{SN}$ PULSE from Cytron Technologies. This pulse sensor operates by emitting light from the green LED to human skin and tissue, and receives the reflected light from the skin and tissue with a photodiode. The pulse sensor interfaces easily with the microprocessor system or microcontroller, which operates with a $5 \mathrm{~V}$ supply and does not require an external circuit in order for the sensor to operate. The pulse sensor has built-in amplification and a noise-cancellation circuit that improves the output signal. The sensor has a single analogue output and the output voltage range is between $0 \mathrm{~V}$ to $5 \mathrm{~V}$, where the output signal amplitude is dependent on the light reflected or absorbed by the skin and tissue, while the sensor is attached to a human finger.

The analogue output from the pulse sensor is converted into 8-bit digital output data, and sent to the microprocessor system by the ADC0804 IC. It interfaces with a microprocessor using an 8-bit parallel data port. The ADC0804 is selected due to the fact that it is 8-bit microprocessor-compatible and it is able to receive an analogue-input voltage range that is the same as the analogue output of the pulse sensor. The circuit configuration of the ADC0804 is set in a free running configuration. Fig. 7 shows the free running configuration of the ADC0804 for this work. In this configuration, an $\mathrm{RC}$ circuit that consists of a
$150 \mathrm{pF}$ capacitor and a $10 \mathrm{~K}$ ohm resistor is used as a clocking circuit for the ADC0804, which allows the ADC to use selfclocking instead of an external clock. With this configuration, the analogue input is converted into a digital output automatically by the internal clock. A conversion time of approximate $100 \mu \mathrm{s}$ is required for the analogue input to be converted into 8-bit digital data successfully [6].

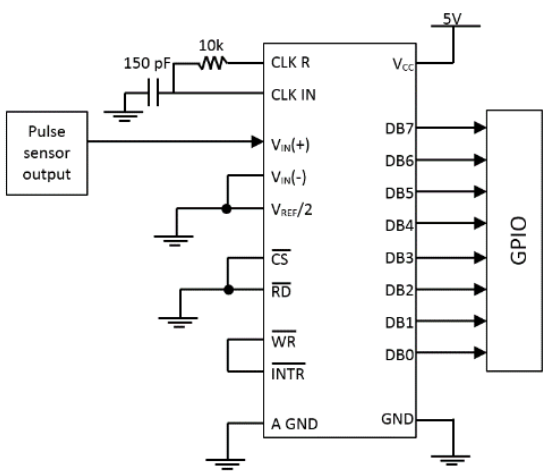

Fig. 7. The free running configuration of the ADC0804.

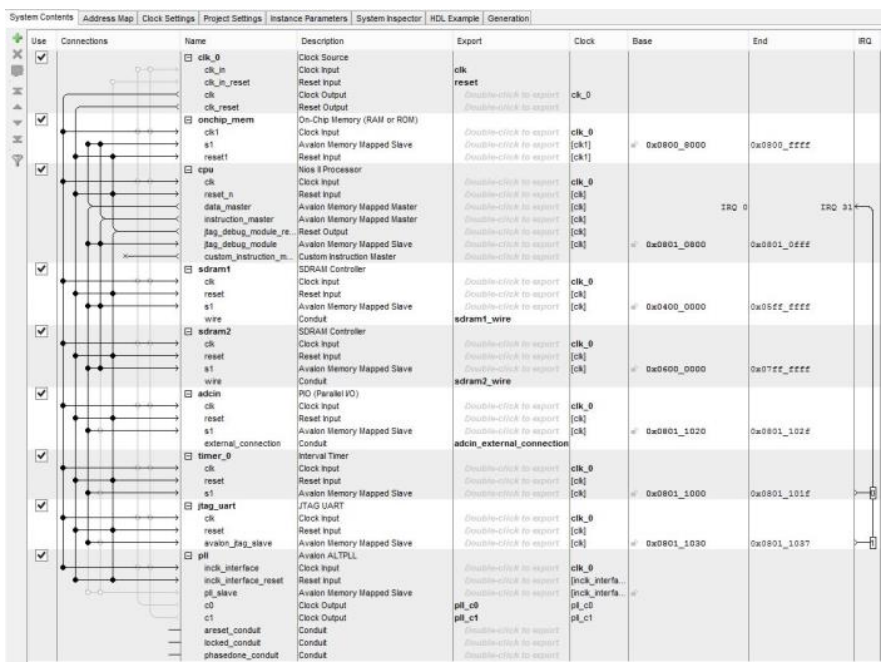

Fig. 8. System interconnection of the proposed microprocessor system in Qsys

The microprocessor system's design is developed by integrating the modules or components together, using the Qsys tool from the Quartus II software. The system's design is compiled and synthesized by using Quartus II software. Its I/O contact is assigned to the system design by Quartus II based on the Cyclone II EP2C70F896C7 FGPA chip. The synthesized system design is downloaded to a Cyclone II EP2C70F896C7 FGPA chip in the Altera DE2-70 Board by using the Quartus II programmer. Fig. 8 shows the system interconnection of the proposed microprocessor system in the Qsys tool. The system interconnection is based on the block diagram of the microprocessor system illustrated in Fig. 6. In this system, a phase-locked loop (PLL) module is used to provide two 50 $\mathrm{MHz}-0.3 \mathrm{~ns}$ shifted clocked pulses to two 32Mb SDRAMs.

The Nios II Software Build Tool is a tool chain for Nios II processor where it is used to compile an application program for the processor, provide a driver and hardware abstraction layer (HAL) support to the processor and download the application's program-to-program memory of the 
microprocessor system. In this work, the application program is developed based on the heart-rate monitoring algorithm and data acquisition system. In the application program, a timer interrupt is activated to perform periodic sampling on the analogue input from the pulse sensor by receiving 8-bit digital output data from the ADC, and storing the data on the SDRAM 1. Since the timer interval period is $500 \mu \mathrm{s}$, the sampling rate of the microprocessor system is $2 \mathrm{kS} / \mathrm{s}$. By setting the timer interrupt, the microprocessor system will sample the data consistently. Once a certain amount of data is stored (1000 data points), the heart-rate monitoring algorithm is carried out by the microprocessor system and the results, such as an inter-beat interval (IBI) and beats per minute (bpm), are stored in the SDRAM 2. At same time, the results are shown in the Nios II console through JTAGUART.

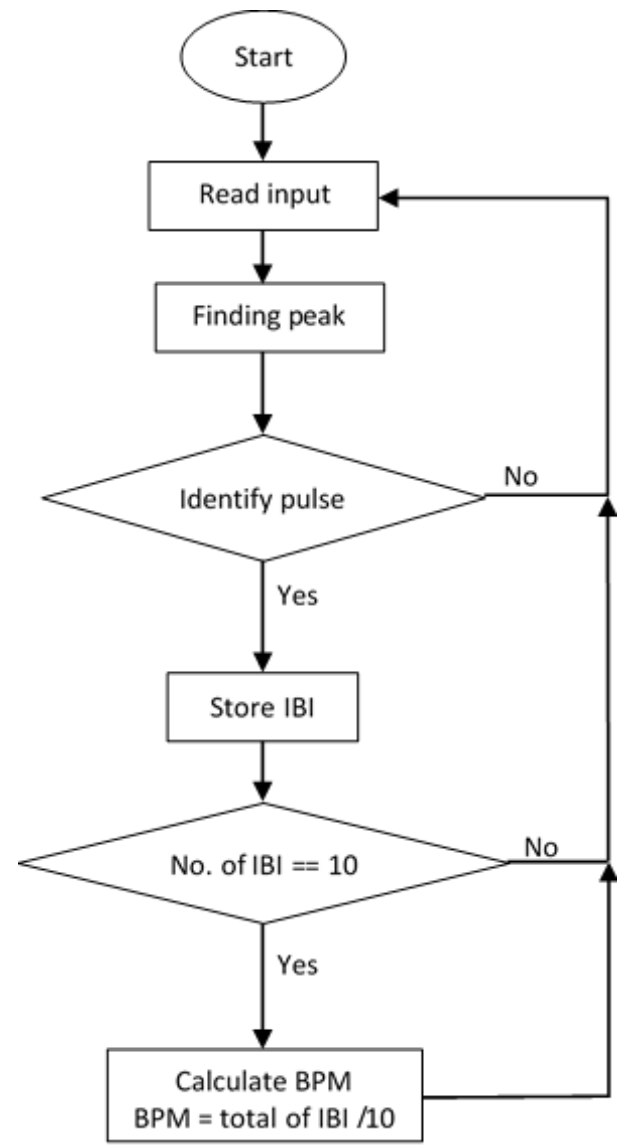

Fig. 9. Flow chart of the beat-finding algorithm for heart-rate monitoring.

The algorithm used for heart-rate monitoring is a beatfinding algorithm. The beat-finding algorithm is a peak detection-based algorithm and the algorithm is based on a "PULSE SENSOR AMPED" website [7]. Fig. 9 shows a simplified flow chart of the beat-finding algorithm for the microprocessor system. The algorithm starts by reading the input data from the SDRAM 1. A peak-finding process will be carried out on the data read from the SDRAM 1. By using the detected peaks, a pulse can be identified based on amplitude of the peaks and the duration between the peaks. The process is repeated until a correct pulse is determined. Once the correct pulse is identified, the IBI is calculated by measuring a period of the pulse between the two nearest peaks and the IBI value is stored in the SDRAM 2. When 10 IBI values are stored, the
BPM is calculated by dividing the total of the IBI value by 10 (Total IBI / 10) and the BPM is stored on the SDRAM 2. In the Nios II console, IBI and BPM values are presented. Fig. 10 shows the pseudocode for the beat-finding algorithm.

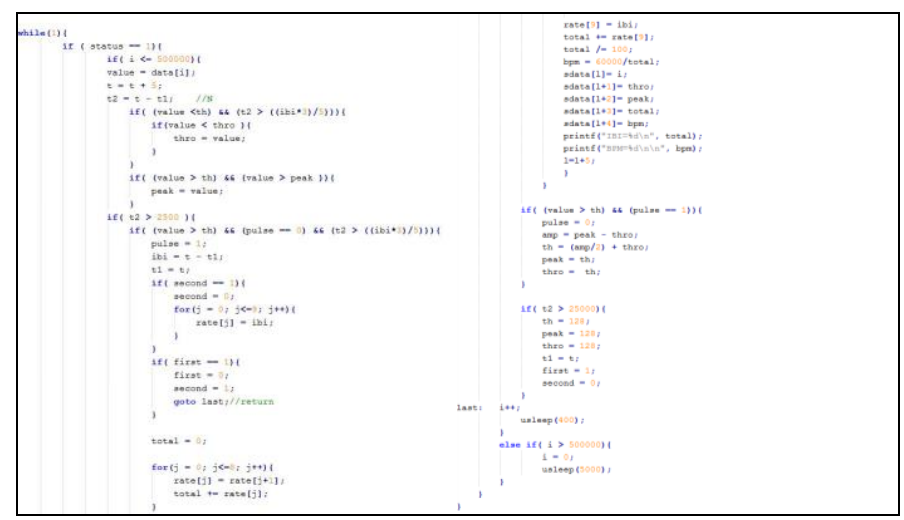

Fig. 10. Pseudocode.

An experiment is set-up to test the functionality of the microprocessor system and beat-finding algorithm. The microprocessor system's design is loaded onto the FPGA board. The FPGA board with an ADC and a pulse sensor is illustrated in Fig. 11. A 25-year-old male student is used as the test target for the heart-rate monitoring. In this experiment, the pulse sensor is attached to the finger for heart-rate measurement.

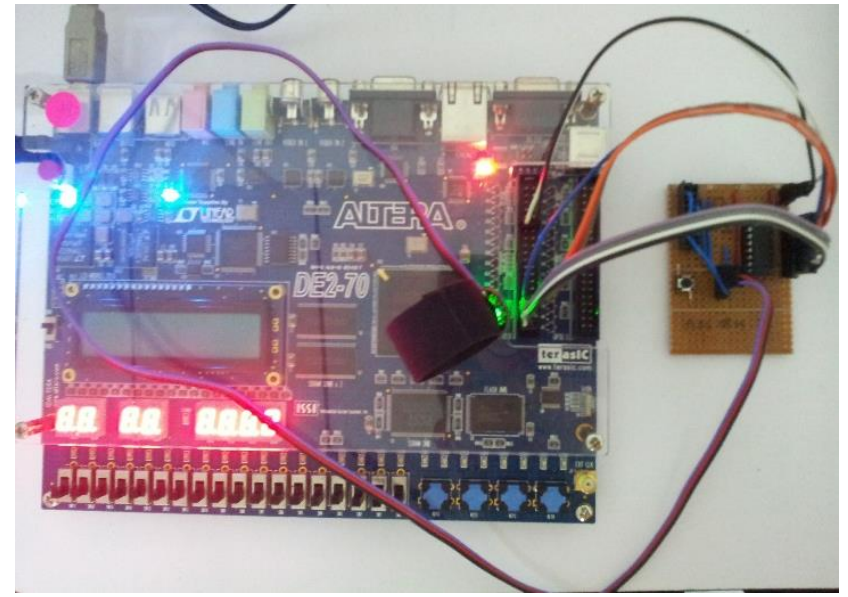

Fig. 11. Microprocessor system in the FPGA board with an ADC and a pulse sensor.

\section{RESUlTS AND DisCUSSION}

In this section, the results of an experiment with the heartrate monitoring system are observed and discussed. In addition, the signal flow within the microprocessor system is discussed in detail. An experiment is set-up to measure the heart rate of a 25-year-old male student, with the pulse sensor attached to a finger. The student is in a resting position (sitting on a chair). From the experiment, the results show that the bpm range is between $68 \mathrm{bpm}$ and $82 \mathrm{bpm}$. This is within the range of the average resting heart rate, which is between $60 \mathrm{bpm}$ to $100 \mathrm{bpm}$. This proved that the heart-rate monitoring system is working as intended. In Fig. 12, the IBI and the bpm values are presented in the Nios II console. 


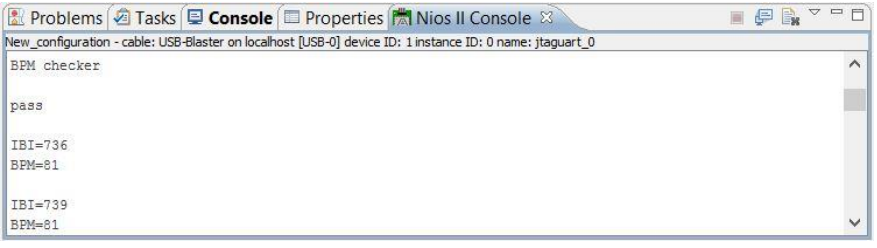

Fig. 12. Nios II console presenting the IBI and bpm values of the target male student.

In order to observe the signal flow in the microprocessor system, SignalTap II from the Quartus II software is used to capture the data transfer within the microprocessor system. SignalTap II is an embedded logic analyser that captures and displays signals in the circuit design. In SignalTap II, all the inputs and outputs are probed to capture the signal flow. The inputs and outputs are the interfaces between the microprocessor and the SDRAMs, and the interfaces between the microprocessor and the $\mathrm{ADC}$, repectively. The clock source for the signal tap is set using the $50 \mathrm{MHz}$ clock source from the DE2-70 board. The data-sample depth is $8 \mathrm{k}$, and the storage qualifier is set as a transitional type. In this embedded logic analyser, three situations are selected to observe the signal flows that are storing data from the ADC to the SDRAM 1 (DRAM0), reading data from the SDRAM 1 and storing the resulting data to the SDRAM 2 (DRAM1). The waveform in Fig. 13, Fig. 14 and Fig. 15 are the captured data obtained by using the SignalTap II embedded analyser in real time. Fig. 13 shows the write operation on the SDRAM 1 by storing the data obtained from the ADC. The green box in Fig. 13 shows the write operation on the SDRAM 1 with data from the ADC output. In Fig. 13, the ADC output (ADCIN) value is $7 \mathrm{DH}$. When the write operation on the SDRAM 1 is carried out, the write enable signal (DRAMO WE N), the column address strobe (DRAMO_CAS_N) and the chip select signal (DRAMO_CS_N) are set to low, while the row address strobe (DRAM0_RAS_N), clock enable (DRAMO_CKE) and system clock input (DEAMO $\quad$ CLK) are set to high [8] bus (DRAMO_A and DRAMO BA), Data I/O mask (DRAMO_LDQMO and DRAMO_UDQM1) and data bus (DRAM $\bar{D} Q$ ) being set, the data are written onto the SDRAM [8].

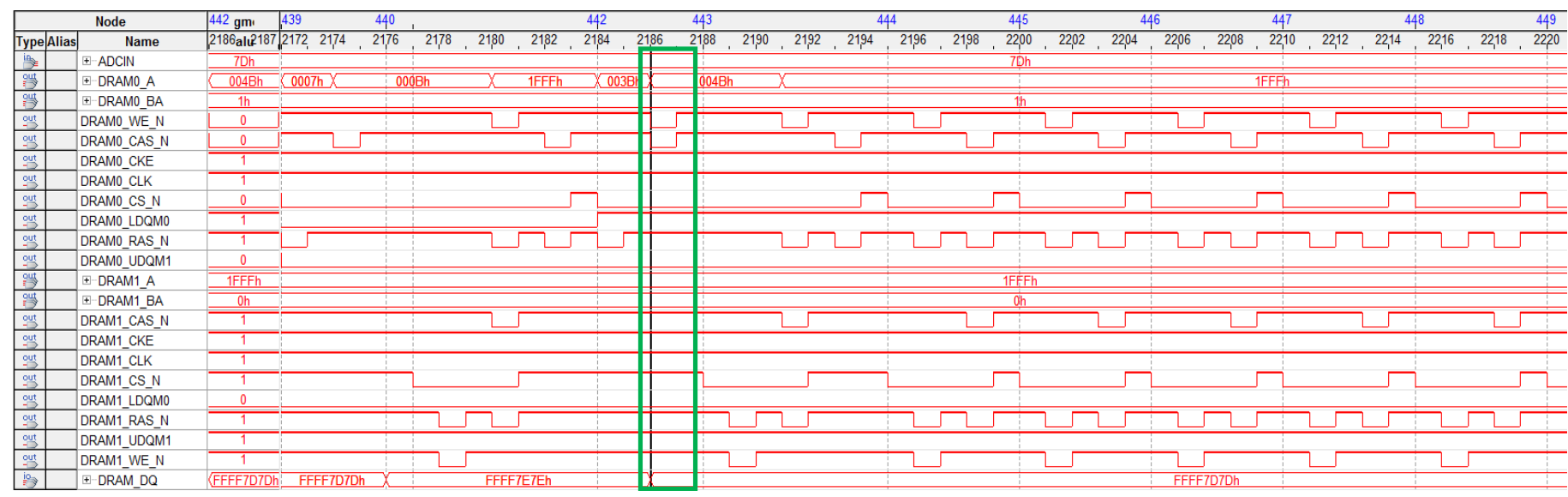

Fig. 13. Captured waveform of storing data from ADC output to SDRAM 1 using SignalTap II

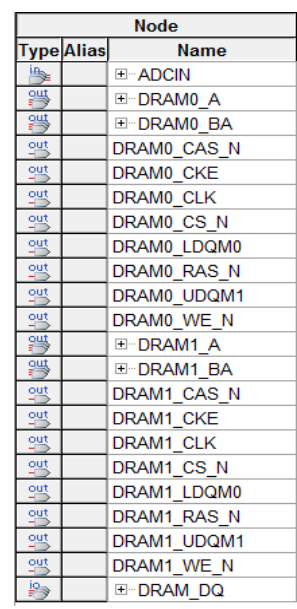

+DRAM_DQ

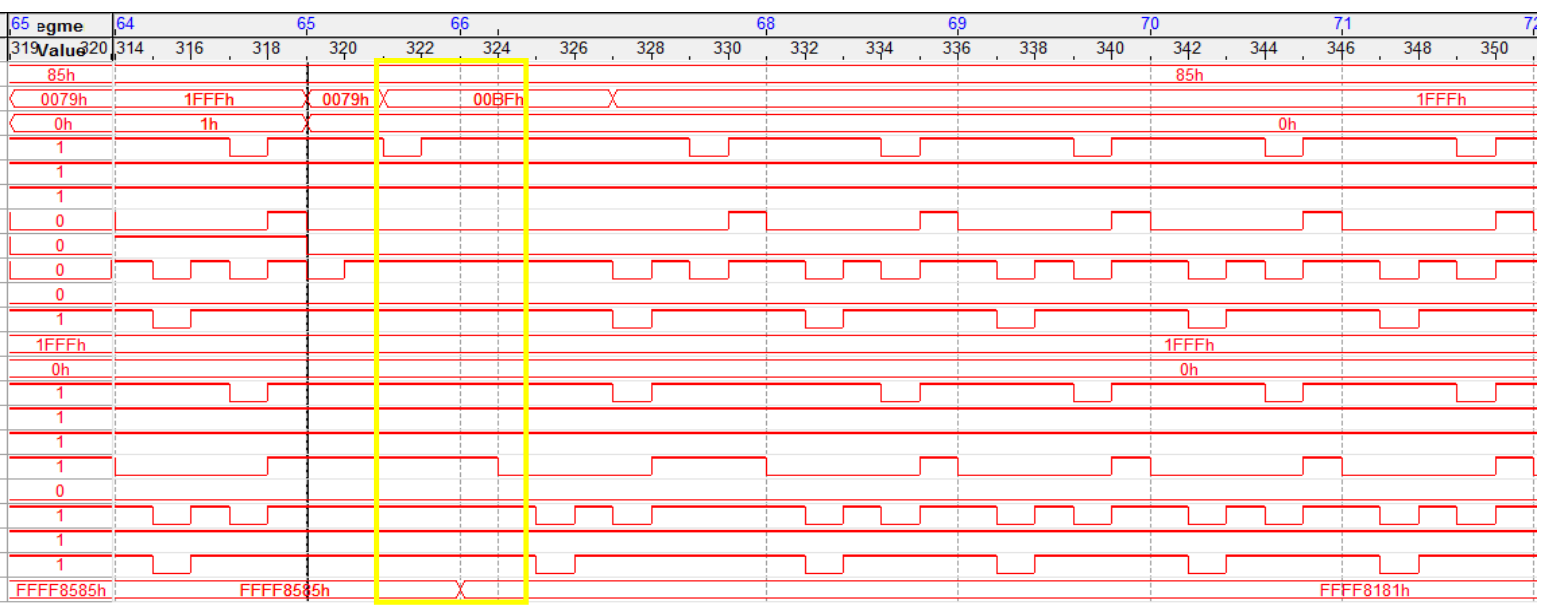

Fig. 14. Captured waveform of reading data from the SDRAM 1 by using SignalTap II 


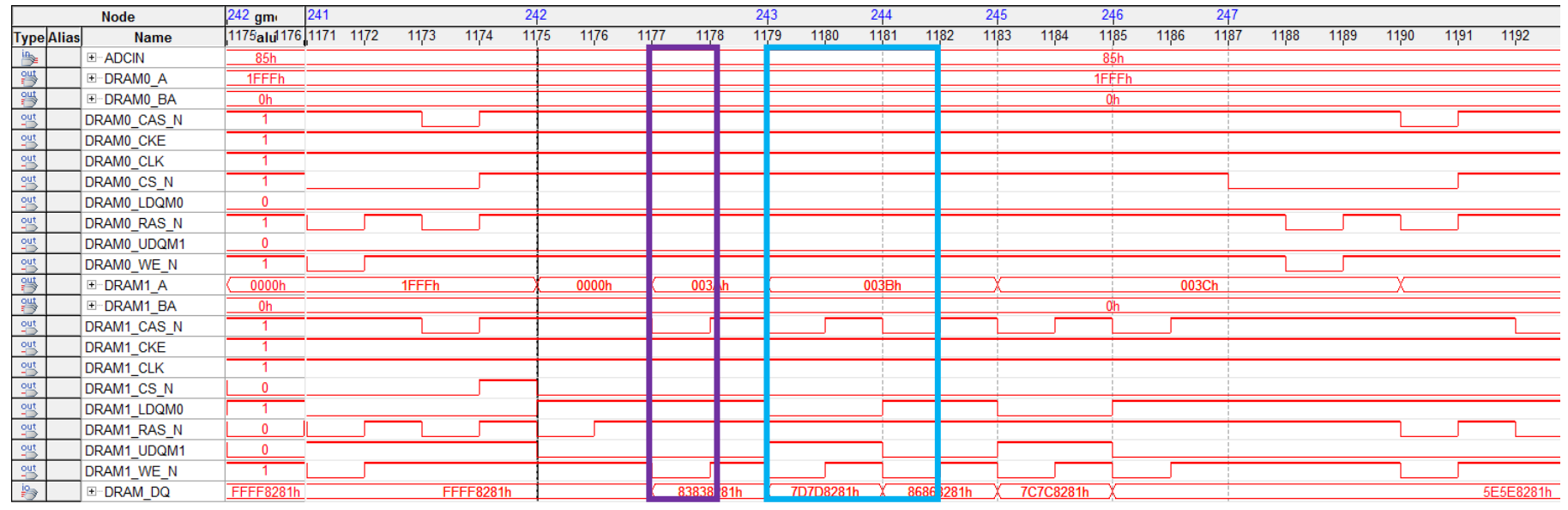

Fig. 15. Captured waveform of storing resulting data to the SDRAM 2 by using SignalTap II

In Fig. 14, the captured waveform of the read operation on the SDRAM 1 is presented. The yellow box in Fig. 14 shows the 8-bit read operation on the SDRAM 1 with the address of $00 \mathrm{BFH}$ to the obtained data value of $81 \mathrm{H}$ (since DRAM DQ is a 32-bit bus, the two least significant bytes are the data value). The read operation is similar to the write operation explained previously, but with the difference that the write enable signal (DRAMO WE N) remains high [8]. In this read operation, there is a latency delay after the write operation has been carried out. The purpose of reading data from the SDRAM 1 is to allow the algorithm to run on the data. In Fig. 15, the captured waveform presents the write operation on data storing in the SDRAM 2 (DRAM1). The purple box shows the write operation of data $83 \mathrm{H}$ on the SDRAM 2 with the address of 003AH (since DRAM DQ is a 32-bit bus, the fifth and sixth byte from the least significant byte are the data values for the SDRAM 2). The blue box shows the write operation of data value $7 \mathrm{D} 86 \mathrm{H}$ on the SDRAM 2 with the address 003BH. From the purple box in Fig. 15, the write operation is carried out twice with the data I/O mask bus (DRAM1 LDQMO and DRAM1 UDQM1) being toggled. This is to allow the high byte value being stored in the SDRAM 2 before the lower byte value is stored. The writing operation in Fig. 15 shows that the resulting data are stored to the SDRAM 2.

TABLE II

TOtal LOGIC ElEMENTS AND POWER DisSIPATION FOR THE PROPOSED MICROPROCESSOR SYSTEM

\begin{tabular}{|c|c|c|c|}
\hline $\begin{array}{c}\text { Total } \\
\text { Logic } \\
\text { Elements }\end{array}$ & $\begin{array}{c}\text { Total } \\
\text { Thermal } \\
\text { Power } \\
\text { Dissipation }\end{array}$ & $\begin{array}{c}\text { Core Static } \\
\text { Thermal } \\
\text { Power } \\
\text { Dissipation }\end{array}$ & $\begin{array}{c}\text { I/O Thermal } \\
\text { Power } \\
\text { Dissipation }\end{array}$ \\
\hline 4,995 & $218.26 \mathrm{~mW}$ & $155.03 \mathrm{~mW}$ & $49.53 \mathrm{~mW}$ \\
\hline
\end{tabular}

Based on the compilation report, the total number of logic elements for the proposed microprocessor system is around 4,995. From the PowerPlay Power Analyzer report, the total thermal power dissipation for the proposed system is 218.26 $\mathrm{mW}$, where the core static thermal power dissipation is around $155.03 \mathrm{~mW}$ and the $\mathrm{I} / \mathrm{O}$ thermal power dissipation is 49.53 $\mathrm{mW}$. Table II tabulates the total logic elements and the power dissipation for the proposed system with different timer module periods. The PowerPlay Power Analysis is carried out using $12.5 \%$ of the default toggle rate for the input I/O signal, and the toggle rates of the remaining signals are vectorless estimated.

\section{CONCLUSION}

In this paper, a microprocessor system loaded with a heartrate measurement algorithm is implemented. The microprocessor system is implemented using FPGA hardware and interfaces with an ADC and a pulse sensor. In a literature review, several existing microprocessor systems using a pulse signal-based sensor are discussed. An experiment is carried out to analyse the functionality of the microprocessor system loaded with the algorithm. The results show that the heart rate detected is within the range of a normal human heart rate. The signal flow of the microprocessor system is observed and analysed. Based on the power analysis results, the proposed microprocessor system has a total power dissipation of around $218.26 \mathrm{~mW}$. In future work, the heart-rate monitoring system will be fabricated into a portable device that allows real-time heart-rate monitoring.

\section{REFERENCES}

[1] Y. Yang and K. Tang, "A Pulse Oximetry System with Motion Artifact Reduction Based on Fourier Analysis," in IEEE International Symposium on Bioelectronics and Bioinformatics (IEEE ISBB 2014), Chung Li, 2014, pp. 1-4.

[2] G. Guo, Z. Li, and F. Yang, "Design of High Speed Pulse Data Acquisition System Based on FPGA and USB," in 2011 International Conference on Multimedia Technology, Hangzhou, 2011, pp. 5374-5376.

[3] H. Liu, Y. Wang, and L. Wang, "FPGA-based Remote Pulse Rate Detection Using Photoplethysmographic Imaging," in 2013 IEEE International Conference on Body Sensor Networks (BSN 2013), Cambridge, 2013, pp. 1-5.

[4] P. An and M. Zeng, "Pulse Monitoring System Based on Feedback Algorithm," in 2012 10th World Congress on Intelligent Control and Automation, Beijing, 2012, pp. 4211-4214.

[5] Q. Gong, G. Li, P. Sun, and Y. Pang, "Design and Implementation of Pulse Signal Detection System Based on Bluetooth Transmission, ” Int. J. Control Autom.., Vol. 8, No. 7, 2015 , pp. 141-148.

[6] "8-Bit uP Compatible A/D Converters," ADC0801/ADC0802/ADC0803/ADC0804/ADC0805 datasheet, National Semiconductor Corp., December 1994

[7] "Pulse Sensor Amped," 2015. [Online]. Available: http://pulsesensor.com/pages/pulse-sensor-amped-arduino-v1dot1.

[8] "256-MBIT SYNCHRONOUS DRAM," IS42S83200B datasheet, Integrated Silicon Solution, Inc., 2007. 